\title{
Environmentally Robust MEMS Vibratory Gyroscopes for Automotive Applications
}

\author{
Cenk Acar, Member, IEEE, Adam R. Schofield, Member, IEEE, Alexander A. Trusov, Student Member, IEEE, \\ Lynn E. Costlow, Member, IEEE, and Andrei M. Shkel, Senior Member, IEEE
}

\begin{abstract}
Automotive applications are known to impose quite harsh environmental conditions such as vibration, shock, temperature, and thermal cycling on inertial sensors. Micromachined gyroscopes are known to be especially challenging to develop and commercialize due to high sensitivity of their dynamic response to fabrication and environmental variations. Meeting performance specifications in the demanding automotive environment with low-cost and high-yield devices requires a very robust microelectromechanical systems (MEMS) sensing element. This paper reviews the design trend in structural implementations that provides inherent robustness against structural and environmental parameter variations at the sensing element level. The fundamental approach is based on obtaining a gain and phase stable region in the frequency response of the sense-mode dynamical system in order to achieve overall system robustness. Operating in the stable sense frequency region provides improved bias stability, temperature stability, and immunity to environmental and fabrication variations.
\end{abstract}

Index Terms-Automotive sensors, inertial sensors, microelectromechanical systems (MEMS), micromachined gyroscopes.

\section{INTRODUCTION}

A UTOMOTIVE gyroscopes are highly engineered inertial sensors, involving a large number of disciplines including microelectromechanical systems (MEMS) technology and micromachining, application-specific integrated circuit (ASIC) and semiconductor technology, analog and digital signal processing, electronic networking interfaces, packaging and electronics integration, and high-volume manufacturing techniques. The automotive environment imposed on these devices is a daunting combination of temperature, vibration, thermal cycling, shock, humidity, acoustic, etc. Performance specifications must be met by accurately detecting angstroms of Coriolis-induced motion during or after exposure to these environments. In addition, the subject requires intellectual

Manuscript received August 20, 2008; revised February 18, 2009. Current version published October 28, 2009. This work was supported in part by the BEI Technologies under Contract BEI-36974, in part by the University of California Discovery Program ELE04-10202, and in part by the National Science Foundation under Grant CMS-0409923. The associate editor coordinating the review of this paper and approving it for publication was Prof. Paul Acar.

C. Acar is with Systron Donner Automotive, Schneider Electric, Concord, CA 94518-1399 USA (e-mail: cenkacar@gmail.com).

A. R. Schofield, A. A. Trusov, and A. M. Shkel are with the University of California, Irvine, CA 92697 USA (e-mail: adam.schofield@uci.edu; atrusov@uci. edu; andrei.shkel@uci.edu).

L. E. Costlow, retired, was with the Systron Donner Automotive, Custom Sensors and Technologies, Schneider-Electric, Concord, CA 94518-1399 USA (e-mail: 1costlow@gmail.com).

Color versions of one or more of the figures in this paper are available online at http://ieeexplore.ieee.org.

Digital Object Identifier 10.1109/JSEN.2009.2026466

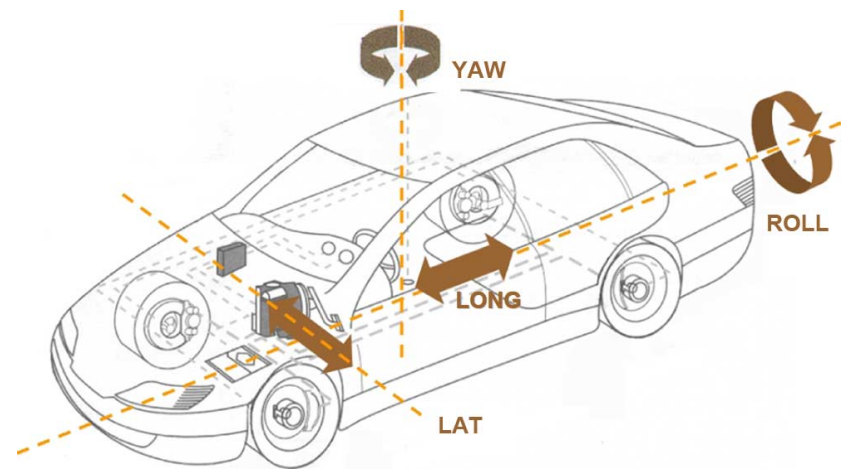

Fig. 1. Illustration of the angular rate and acceleration detection axes in automotive applications. Majority of emerging safety and comfort systems require yaw- and roll-rate sensing.

property concepts capable of the robustness required to efficiently produce the gyros and a huge investment to fund years of development and production line installation prior to production launch.

Gyroscopes typically operate in safety systems, where high reliability and quality must approach the zero parts per million level. Detection of sensor failures is of utmost importance to avoid an unintended vehicle action that endangers people. An automotive gyroscope must meet all these requirements in addition to having extremely small size and low-unit production cost. As with any high-volume technology, prices continue to drop as volumes grow and the market matures.

In this paper, we review the primary automotive applications, specification requirements, and commercial status of MEMS gyroscopes, discuss the fundamental technical challenges in design and high-volume production, and finally, present an overview of the innovative multi-degree of freedom (DOF) gyroscope design trend that aims to achieve inherent robustness at the mechanical sensing element level.

\section{Automotive APPLiCATIONS OF MEMS Gyroscopes}

Several applications for MEMS angular rate sensors have emerged since the mid-1990s in the automotive field. The most significant one has been yaw-rate sensors (Fig. 1) for electronic stability control (ESC) brake systems, which is a major breakthrough in automotive safety. ESC helps prevent accidents by automatically activating asymmetric individual wheel braking actions in an out-of-control (oversteering or understeering) vehicle to stabilize it to regain traction and driver control. The other automotive safety systems application involves roll-rate 
TABLE I

TypicAl Automotive Gyro Key SPECIFICATIONS

\begin{tabular}{|c|c||c|c|c|c|c|}
\hline \multicolumn{2}{|c||}{ SPECIFICATION } & \multicolumn{4}{c|}{ APPLICATION } \\
\hline PARAMETER & UNITS & $\begin{array}{c}\text { NAV } \\
\text { GPS } \\
\text { BACKUP }\end{array}$ & $\begin{array}{c}\text { ROLLOVER } \\
\text { AIRBAG } \\
\text { DEPLOY }\end{array}$ & $\begin{array}{c}\text { ESC } \\
\text { Passenger } \\
\text { Compartment }\end{array}$ & $\begin{array}{c}\text { ESC } \\
\text { Engine } \\
\text { Compartment }\end{array}$ & $\begin{array}{c}\text { LANE } \\
\text { KEEPING }\end{array}$ \\
\hline Motion Sense & Axis & Yaw & Roll & Yaw & Yaw & Yaw \\
\hline Op Temp Range & ${ }^{\circ} \mathrm{C}$ & -40 to +85 & -40 to +85 & -40 to +85 & -40 to +125 & -40 to +105 \\
\hline Rate range & ${ }^{\circ} / \mathrm{s}$ & \pm 75 & \pm 300 & \pm 75 to \pm 100 & \pm 75 to \pm 100 & \pm 75 \\
\hline Scale Factor Error & $\%$ Nom SF & \pm 5 & \pm 5 & \pm 3 & \pm 3 & \pm 1 \\
\hline Total Offset & ${ }^{\circ} / \mathrm{s}$ & \pm 5 & \pm 5 & \pm 3 & \pm 3 & \pm 2 \\
\hline Offset over Temp Range & $\circ / \mathrm{s}$ & \pm 10 & \pm 5 & \pm 2 & \pm 2 & \pm 0.5 \\
\hline Offset Drift Velocity & $\% / \mathrm{s} / \mathrm{min}$ & \pm 5 & \pm 5 & \pm 3 & \pm 3 & \pm 2 \\
\hline
\end{tabular}

gyroscopes to sense impending vehicle rollover conditions, for deployment of passenger protection airbags and even preventing rollover as a part of the ESC system.

Applications outside safety systems also exist, such as yaw-rate gyros used to support backup navigation systems by dead reckoning to preserve GPS-based navigator function during temporary loss of GPS signal. Gyroscopes are enabling new higher end applications as well. For example, an emerging application is lane-keeping systems that may require high-precision detection of yaw rate. These systems assist the driver's steering action to preserve the vehicle in the existing highway lane. Lane-keeping assistance systems can make minor steering corrections if the vehicle detects an imminent lane departure without a driver-activated turn signal.

\section{A. Typical Automotive Gyro Specifications}

Table I presents typical key specifications for automotive gyros and compares required performance specifications for each application. Until recently, ESC posed the most stringent offset (bias), offset over temperature and scale factor error specifications for automotive MEMS gyros. With the trend to mount ESC gyros in the engine compartment, the operation temperature range increased drastically. However, the emerging lane-keeping applications have even tighter specifications. Rollover airbag sensors and GPS backup sensors are among the lower end performance applications.

\section{B. Commercial MEMS Automotive Gyroscopes}

The strict performance specifications and cost requirements in the automotive insdustry have resulted in only five companies worldwide that share the ESC market as of 2008. Some of them also have various positions in the rollover, navigation, and lane-keeping gyro markets. These automotive ESC gyroscope suppliers take very different design and technology approaches to the implementation of their micromachined gyroscopes. These five companies have a widely divergent share of the ESC market. Table II depicts the market share and MEMS technology utilized by these ESC gyro suppliers in the 2006 market [2].

One of the automotive gyroscopes that holds a significant market share is quartz rate sensor (QRS) from Systron Donner Automotive introduced in 1996 and now in its third generation [3]. Its mechanical structure consists of a double-ended tuning fork operated at or above $10 \mathrm{kHz}$ with separate drive and sensing tines optimized for their respective drive and sense functions, utilizing piezoelectric actuation and detection. Masses on the
TABLE II

LEADING Automotive ESC GyRo SuPPLIERS [2]

\begin{tabular}{|l|c|l|l|}
\hline SUPPLIER & $\begin{array}{c}\text { Worldwide } \\
\text { Market Share } \\
(2006)\end{array}$ & $\begin{array}{l}\text { MEMS } \\
\text { Micromachining } \\
\text { Technology }\end{array}$ & $\begin{array}{l}\text { Drive/Sense } \\
\text { Technique }\end{array}$ \\
\hline Systron Donner & $43 \%$ & Quartz wet etch & Piezo / piezo \\
\hline Bosch & $34 \%$ & Silicon bulk DRIE & Cap. / cap. \\
\hline Silicon Sensing & $11 \%$ & Silicon bulk DRIE & Ind. / ind. \\
\hline Panasonic & $9 \%$ & Quartz wet etch & Piezo / piezo \\
\hline Analog Devices & $3 \%$ & Silicon surface & Cap. / cap. \\
\hline
\end{tabular}

tine ends are laser trimmed to automatically adjust resonant frequencies and quadrature error [4].

As for silicon devices, Robert Bosch GmbH introduced a micromachined yaw sensor, the DRS-MM1, in 1998 which is now in its third generation [6]. The mechanical design consists of two coupled $11 \mu \mathrm{m}$ thick polysilicon masses forming a tuning fork structure with decoupled drive and detection frames that is operated at approximately $15 \mathrm{kHz}$ [7]. Both drive and sense are operated closed loop using a digital ASIC, which also adjusts the output to compensate for both offset and scale factor temperature drifts [8].

A relatively new device to the automotive market is the Analog Devices fully integrated gyroscope ADXRS (also currently in its third generation), which was put into mass production in 2002 after four years in preproduction development [5], [9]. The mechanical sensor consists of two (or up to four) mechanically independent $4-\mu \mathrm{m}-$ thick polysilicon structures operated differentially at $15 \mathrm{kHz}$ with a quality factor of 45; the Coriolis signal is compensated using a temperature-variable amplifier, resulting in sensitivity variations of $1 \%$ over a range of $-55{ }^{\circ} \mathrm{C}$ to $85{ }^{\circ} \mathrm{C}$ [10], [11]. Since 2006, Panasonic has moved into silicon tuning fork sensors with a piezoelectric coating material used for both drive and sense. However, there are no publications by Panasonic on the details of their gyroscope design. In addition, Silicon Sensing Systems has commercialized in 2007 a silicon bulk micromachined gyro with capacitive drive and sense, to replace their traditional inductive version eliminating the magnet characteristic [12].

As evidenced by the literature on commercially available gyroscopes, the stability of the output over a broad temperature range is critical for automotive applications. Generally, bias and scale factor temperature drifts are addressed through active signal processing techniques that compensate the output based on the sensed die temperature. However, stability and robustness of the mechanical sensing element becomes crucial 
to meet strict specifications with minimal yield loss. This paper presents a review of an alternative design concept, which aims to passively improve robustness by mechanical design through the use of multi-DOF sense systems [1].

\section{Fundamental Challenges}

The basic sensing element structure of a vibratory gyroscope is composed of a drive oscillator that generates and maintains a constant momentum, and a sense accelerometer that measures the sinusoidal Coriolis force induced due to the combination of the drive vibration and an angular rate input.

Vast majority of reported micromachined rate gyroscopes utilize a vibratory proof mass suspended by flexible beams above a substrate. The primary objective of the dynamical system is to form a vibratory oscillator in the drive mode, coupled to the sense mode accelerometer by the Coriolis force orthogonal to the drive motion and rate input axes.

The drive mode oscillator is most commonly a 1-DOF resonator, which can be modeled as a mass-spring-damper system consisting of the drive proof mass $m_{d}$, the drive-mode suspension system with stiffness $k_{d}$, and the drive damping $c_{d}$. With a sinusoidal drive-mode excitation force, the drive equation of motion along the $x$ axis becomes

$$
m_{d} \ddot{x}+c_{d} \dot{x}+k_{d} x=F_{d} \sin \omega t .
$$

The scale factor of the gyroscope is directly proportional to the drive-mode oscillation amplitude. The phase and the frequency of the drive oscillation directly determines the phase and the frequency of the Coriolis force and thus the sense mode response. Consequently, it is extremely critical to maintain a drive-mode oscillation with stable amplitude, phase, and frequency. In practical implementations, almost all reported gyroscopes operate exactly at resonance, with a phase of $-90^{\circ}$ relative to the drive signal, and the amplitude

$$
x_{0}=Q_{d} \frac{F_{d}}{m_{d} \omega_{d}^{2}}, \quad \text { where } \omega_{d}=\sqrt{\frac{k_{d}}{m_{d}}}, \quad Q_{d}=\frac{m_{d} \omega_{d}}{c_{d}} .
$$

Self-resonance by the use of an amplitude-regulated positive feedback loop is a common and convenient method to achieve a stable drive-mode amplitude and phase. The positive feedback loop destabilizes the resonator and locks the operational frequency to the drive-mode resonant frequency. This allows to set the oscillation phase exactly $90^{\circ}$ from the excitation signal. An automatic gain control (AGC) loop detects the oscillation amplitude, compares it with a reference amplitude signal, and adjusts the gain of the positive feedback to match the reference amplitude. Operating at resonance in the drive mode also minimizes the excitation voltages during steady-state operation.

The sense-mode Coriolis accelerometer is also typically a 1-DOF oscillator, primarily to take advantage of resonance to amplify the mechanical response to the Coriolis force. The equation of motion of the 1-DOF sense-mode oscillator is

$$
m_{s} \ddot{y}+c_{s} \dot{y}+k_{s} y=-2 m_{C} x_{0} \omega_{d} \Omega_{z} \cos \left(\omega_{d} t+\phi_{d}\right)
$$

where $m_{C}$ is the portion of the driven proof mass that contributes to the Coriolis force, and $m_{s}$ is the portion of the proof mass that responds to the Coriolis force. Defining the sensemode resonant frequency $\omega_{s}$ and quality factor ( $Q$ factor) $Q_{s}$, amplitude of the steady-state Coriolis response becomes

$$
y_{0}=\Omega_{z} \frac{m_{C} \omega_{d}}{m_{s} \omega_{s}^{2}} \frac{2 x_{0}}{\sqrt{\left[1-\left(\frac{\omega_{d}}{\omega_{s}}\right)^{2}\right]^{2}+\left[\frac{1}{Q_{s}} \frac{\omega_{d}}{\omega_{s}}\right]^{2}}}
$$

where

$$
\omega_{s}=\sqrt{\frac{k_{s}}{m_{s}}}, Q_{s}=\frac{m_{s} \omega_{s}}{c_{s}} .
$$

To achieve the maximum possible gain in the sense mode, it is generally desired to operate at or near the peak of the sense-mode response curve. This is typically achieved by matching drive and sense resonant frequencies. For a mode-matched system with $\omega_{s}=\omega_{d}$ the sense mode phase is $-90^{\circ}$ relative to the drive velocity, and the amplitude becomes

$$
y_{0 \text { matched }}=\Omega_{z} \frac{2 Q_{s} x_{0} m_{C}}{m_{s} \omega_{s}} .
$$

Even though matching the drive and sense-mode resonant frequencies greatly enhances the sense-mode mechanical response to angular rate input, it comes with many disadvantages. Operating close to the sense resonant peak limits the sensor bandwidth and makes the system very sensitive to variations in system parameters that cause a shift in the resonant frequencies or damping. To illustrate this effect, let us consider a sense mode system with a resonant frequency of $f_{s}=10 \mathrm{kHz}$ and a $Q$ factor of $Q_{s}=10000$. When the operating frequency matches the sense-mode resonant frequency $\omega_{s}$, the amplification factor is 10000 . If there is only $5-\mathrm{Hz}$ relative shift between the drive mode and sense mode frequencies, the scale factor drops by more than $90 \%$.

Given the structural and environmental effects that result in quite large variations in the resonant frequencies, it is extremely difficult to control the drive and sense frequencies with the precision required for mode-matched devices. Thus, it is a common practice to operate away from the resonant frequency of the sense mode, where the frequency variations have reduced effect on the output gain and phase (Fig. 2). This is achieved by setting the sense-mode frequency $f_{s}$ spaced by a certain percentage away from the drive mode frequency $f_{d}$. This frequency separation is commonly defined as $\Delta f=f_{s}-f_{d}$. For higher $\Delta f$ values, the effect of relative frequency variations between the drive and sense modes on the scale factor is reduced (Fig. 3).

In addition to frequency variations, the gain is affected directly by fluctuations in damping, which means that the $Q$ factor of the sense mode response also has to be controlled tightly. Thus, maintaining a constant pressure around the device using a leak-free hermetic packaging is crucial. In addition, damping is also highly temperature dependent. To achieve stable bias and scale factor over temperature, operating away from the resonance peak becomes imperative.

In this paper, we review micromachined gyroscopes with multi-DOF sense-mode that aim to eliminate the limitations of mode-matched or near mode-matched gyroscopes by providing inherent robustness at the sensing element level [1]. The fundamental approach is based on obtaining a flat region instead 

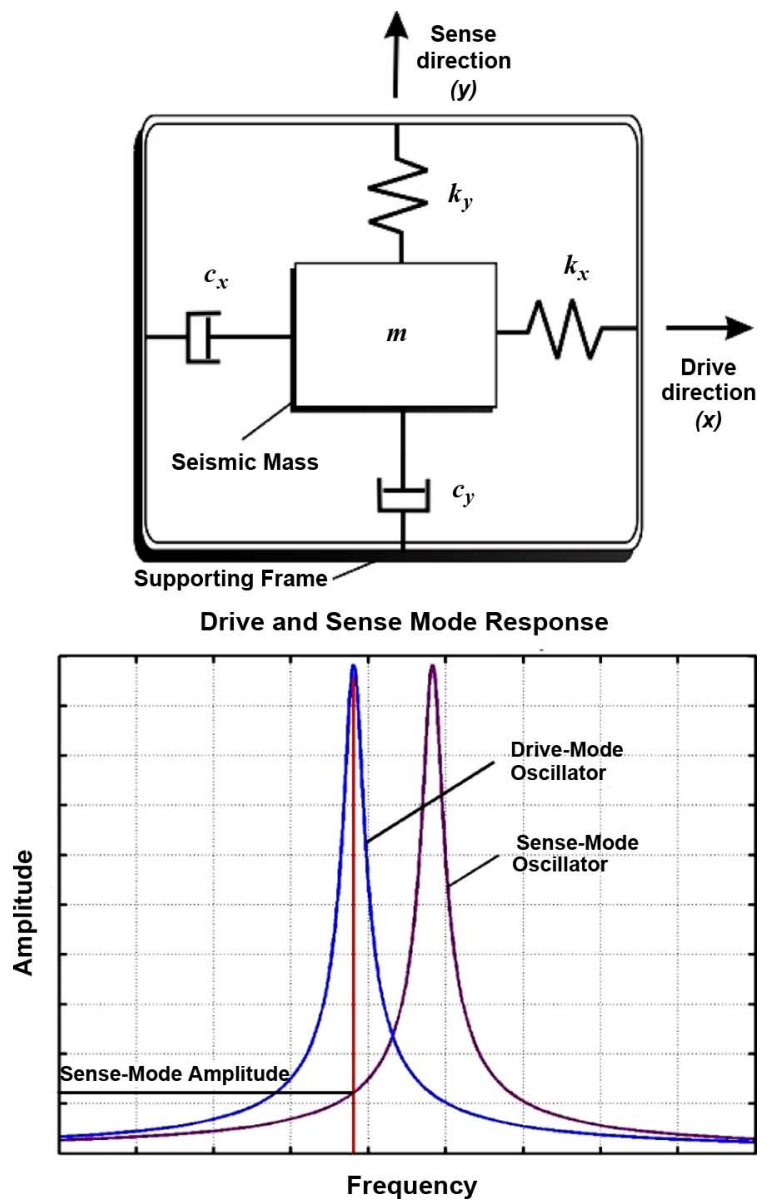

Fig. 2. Vibratory rate gyroscope system is composed of a 1-DOF drive-mode oscillator and a 1-DOF sense-mode oscillator. The drive and sense resonant frequencies are usually spaced by $\Delta f$ to provide robustness.

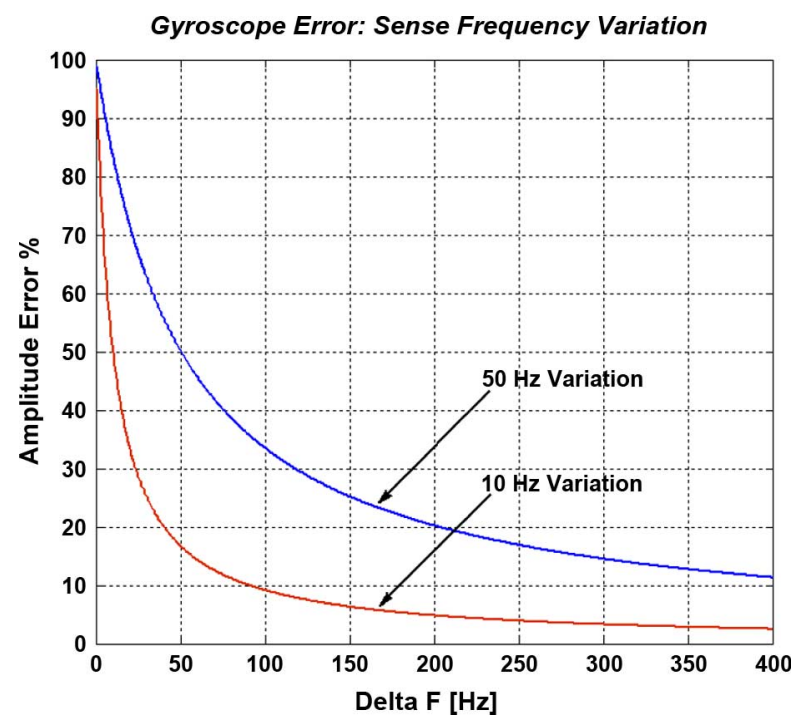

Fig. 3. Increasing $\Delta f$ values reduce the effect of frequency variations on the scale factor.

of a resonant peak in the frequency response of the sense-mode dynamical system. First, the basic concept with a 2-DOF sense mode and a 1-DOF drive mode is presented in Section IV. The antiphase tuning fork gyroscope with multi-DOF sense modes

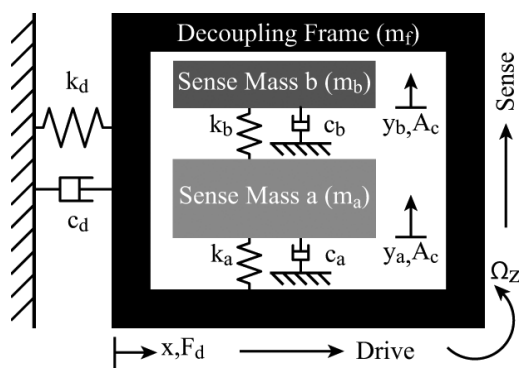

Fig. 4. Lumped structural model of multi-DOF sense-mode gyroscope.

that improves vibration and shock immunity is described in Section V. Finally, a new multi-DOF concept allowing for flexible scaling of parameters to enable operation at high frequencies is presented in Section VI.

\section{Multi-DOF Sense-Mode Gyroscopes}

The multi-DOF sense-mode design approach explores the possibility of achieving a gain and phase stable region in the frequency response of the sense-mode dynamical system. Operating in a stable region addresses some of the major challenges in device robustness:

1) Scale factor variation due to $\Delta f$ variation from die to die and from wafer to wafer.

2) Bias and scale factor variation over temperature due to temperature dependence of $\Delta f$ and sense-mode $Q$ factor.

3) Variation in the Coriolis signal phase due to the shift in natural frequencies.

4) Long-term variation in bias and scale factor due to packaging pressure degradation over time.

The basic concept is based on a 2-DOF sense-mode oscillator in the gyroscope dynamical system, by the use of two interconnected proof masses (Fig. 4 and 6). The two sense masses $m_{a}$ and $m_{b}$ are elastically coupled in the sense direction. The first sense mass $m_{a}$ is free to oscillate both in the drive and sense directions and is excited in the drive direction. In the sense mode, the second sense mass $m_{b}$ is free to move relative to $m_{a}$ and becomes the second DOF. In the drive mode, $m_{a}$ and $m_{b}$ oscillate together and form a resonant 1-DOF oscillator similar to a conventional micromachined gyroscope. With these assumptions, the simplified equations of motion in the drive and sense modes become

$$
\begin{aligned}
\ddot{x}+\frac{c_{d}}{M_{d}} \dot{x}+\frac{k_{d}}{M_{d}} x & =\frac{F_{d}}{M_{d}} \\
\ddot{y}_{a}+\frac{c_{a}}{m_{a}} \dot{y}_{a}+\left(\frac{k_{a}+k_{b}}{m_{a}}\right) y_{a}-\frac{k_{b}}{m_{a}} y_{b} & =-2 \dot{x} \Omega_{z} \\
\ddot{y}_{b}+\frac{c_{b}}{m_{b}} \dot{y}_{b}+\frac{k_{b}}{m_{b}} y_{b}-\frac{k_{b}}{m_{b}} y_{a} & =-2 \dot{x} \Omega_{z} .
\end{aligned}
$$

The 2-DOF sense-mode oscillator provides a frequency response with two resonant peaks and a flat region between the peaks (Fig. 5), instead of a single resonance peak as in conventional gyroscopes. The device is nominally operated in the flat region of the sense-mode response curve, where the gain is less sensitive to variations in the natural frequencies and damping. Thus, reduced sensitivity to structural and thermal 


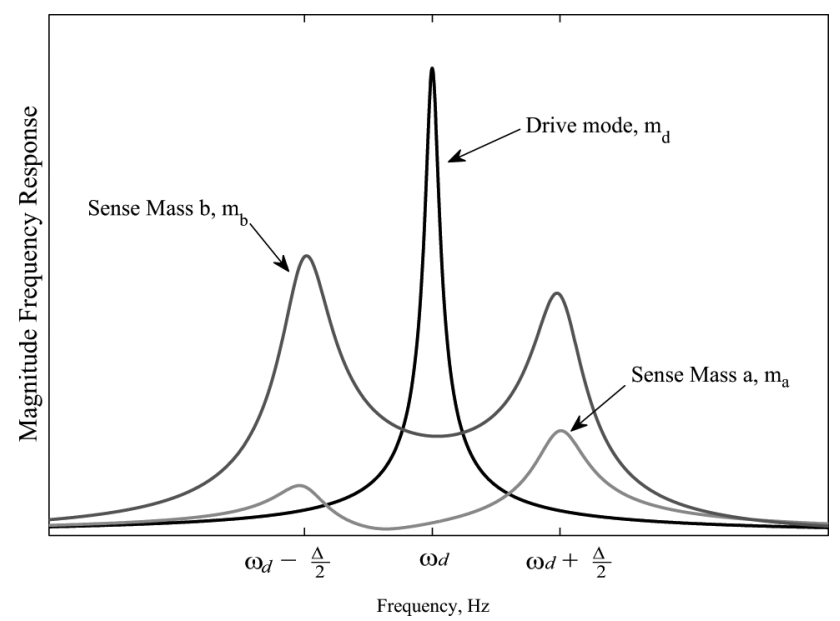

Fig. 5. Frequency response showing superimposed drive and sense modes.

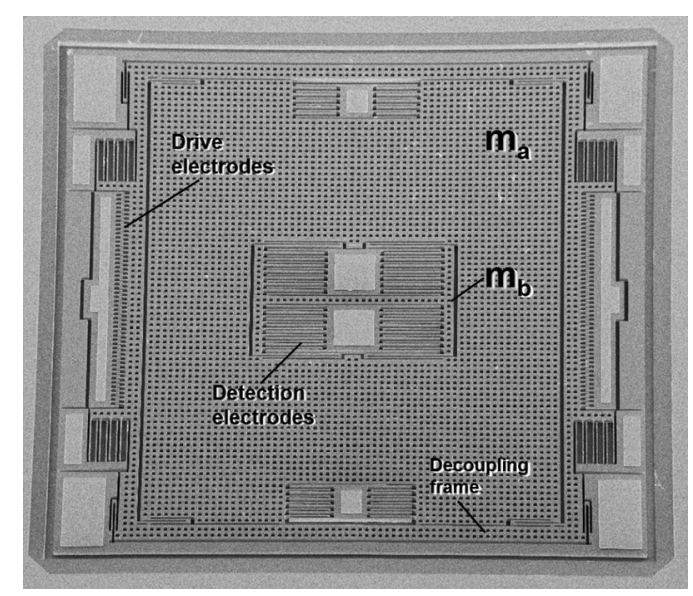

Fig. 6. SEM image of fabricated silicon-on-insulator multi-DOF sense-mode gyroscope.

parameter fluctuations and damping changes are achieved, leading to improved long-term stability and robustness against temperature variations and fabrication variations from device to device [13]-[15].

\section{A. Sense-Mode Design Equations}

As mentioned earlier, multi-DOF sense-mode gyroscopes have an expanded design space compared to conventional devices due to the additional mass forming the 2-DOF sense mode. Thus, the design is characterized by three frequencies: the operational frequency $\omega_{d}$ and the lower and higher coupled sense-mode resonances $\omega_{1}$ and $\omega_{2}$, respectively. The drive mode of a multi-DOF device is a conventional 1-DOF dynamic system, where the natural frequency $\omega_{d}$ can be selected independent of the sense mode by adjusting the drive mode stiffness $k_{d}$ or the decoupling frame mass $m_{f}$ (see Fig. 4). Therefore, the focus of designing a multi-DOF gyroscope is on the 2-DOF sense mode, specifically on identifying the structural parameters $k_{a}, k_{b}, m_{a}$, and $m_{b}$ needed to realize the desired resonant frequencies $\omega_{1}$ and $\omega_{2}$.

The sense-mode resonant frequencies, however, are not independent of the drive mode since the device is designed to take advantage of the constant amplitude region in the sense-mode frequency response. Assuming the drive is equally spaced from the peaks, the desired sense-mode resonant frequencies can be expressed as

$$
\omega_{1,2}=\omega_{d} \mp \frac{\Delta}{2}
$$

where $\omega_{d}$ is the operational frequency, and $\Delta$ is the sense mode peak spacing, defined as $\Delta=\omega_{2}-\omega_{1}$. The three characteristic frequencies of the multi-DOF concept can be completely defined using only two parameters: the operational frequency $\omega_{d}$ and sense-mode peak spacing $\Delta$, as shown in Fig. 5. Solving the inverse eigenvalue problem for the 2-DOF sense mode gives [16]

$$
\begin{array}{r}
k_{a}=m_{a}\left[\frac{\omega_{d}^{2}+\left(\frac{\Delta}{2}\right)^{2}+2 \mu^{2}\left(\omega_{d}^{2}+\left(\frac{\Delta}{2}\right)^{2}\right)}{1+\mu^{2}}\right. \\
\left.\mp \frac{\left.\sqrt{\omega_{d}^{2} \Delta^{2}-\mu^{2}\left(\omega_{d}+\frac{\Delta}{2}\right)^{2}\left(\omega_{d}-\frac{\Delta}{2}\right)^{2}}\right]-k_{b}}{1+\mu^{2}}\right] \\
k_{b}=m_{b}\left[\frac{\omega_{d}^{2}+\left(\frac{\Delta}{2}\right)^{2}}{1+\mu^{2}}\right. \\
\left. \pm \frac{\sqrt{\omega_{d}^{2} \Delta^{2}-\mu^{2}\left(\omega_{d}+\frac{\Delta}{2}\right)^{2}\left(\omega_{d}-\frac{\Delta}{2}\right)^{2}}}{1+\mu^{2}}\right]
\end{array}
$$

which are the sense-mode design equations for the suspensions required to achieve the desired resonant frequencies in terms of the drive frequency $\omega_{d}$, peak spacing $\Delta$, and mass ratio $\mu^{2}=m_{b} / m_{a}$.

Examination of (11) and (12), however, reveals the existence of a constraint for physically achievable systems

$$
\omega_{d}^{2} \Delta^{2}-\mu^{2}\left(\omega_{d}+\frac{\Delta}{2}\right)^{2}\left(\omega_{d}-\frac{\Delta}{2}\right)^{2}>0 .
$$

Therefore, combinations of mass ratios, peak spacings, and operational frequencies cannot be selected arbitrarily, but rather must be chosen to ensure (13) is met. This gives rise to two methods of operational frequency scaling and associated tradeoffs, which is discussed in more detail in [16].

\section{B. Sense-Mode Gain and Bandwidth}

The mechanical gain and bandwidth of a multi-DOF sense mode gyroscope are dependent on the amplitude of the region between the peaks of the 2-DOF sense-mode frequency response. Despite the presence of two sense masses, the mechanical parameters of the device are only dependent on the magnitude of the smaller mass $m_{b}$, as this is the mass used for detection. The reason for this is the location of the antiresonance frequency of the larger mass $m_{a}$, which is always between the sense-mode resonant frequencies, as illustrated in Fig. 5. Therefore, the amplitude of the smaller mass $m_{b}$ is maximized relative to the larger mass between the peaks making it the optimum mass for sensing angular rate induced motion. 
The sense mode gain can be found using the absolute value of the mass $m_{b}$ transfer function at the operational frequency $G_{b}\left(\omega_{d}\right)$, which gives

$$
G_{b}\left(\omega_{d}\right)=\frac{\omega_{d}^{2}+\frac{\Delta^{2}}{4}}{\left(\omega_{d}^{2}-\frac{\Delta^{2}}{4}\right) \Delta^{2}} .
$$

If the peak spacing is assumed to be much smaller than the operational frequency $\omega_{d} \gg \Delta$, which is typical for most 2-DOF systems, (14) reduces to

$$
G_{b}\left(\omega_{d}\right) \approx \frac{1}{\Delta^{2}} .
$$

Thus, (15) shows that the major parameter affecting the sense mode gain of a multi-DOF sense-mode gyroscope is the peak spacing, where a smaller spacing ultimately results in larger gain values independent of operational frequency.

If we assume that the drive motion is a regulated constant amplitude sinusoid with an amplitude $x_{0}$, as in a typical gyroscope system, the sense-mode response amplitude for an angular rate input $\Omega_{z}$ becomes

$$
y_{0} \approx \frac{2 x_{0} \omega_{d}}{\Delta^{2}} \Omega_{z} .
$$

1) Scale Factor Comparison With Conventional Approach: It is clear that the 2-DOF sense-mode approach sacrifices sense mode gain by operating away from resonance. However, it should be remembered that vast majority of conventional devices in practice are mode mismatched, and operated off-resonance in the sense mode to minimize scale factor and bias variation from device to device and over temperature. Thus, a fair comparison can only be made with a mode-mismatched conventional gyroscope.

As an example, let us consider a conventional gyroscope system operated at the drive resonant frequency of $2 \mathrm{kHz}$, with a drive amplitude of $10 \mu \mathrm{m}$. For $1^{\circ} / \mathrm{s}$ angular rate input, with a sense-mode $\mathrm{Q}$ factor of 100 , the sense-mode response amplitude of a mode-matched system, i.e., $\Delta f=0 \mathrm{~Hz}$, is $2.778 \mathrm{~nm}$. Even though increasing the $\Delta f$ in a conventional system improves robustness, it also decreases the scale factor. For example, when the $\Delta f$ is increased to $100 \mathrm{~Hz}$ by setting the sense mode frequency to $2100 \mathrm{~Hz}$, the sense mode amplitude drops to $0.266 \mathrm{~nm}$. This translates into a scale factor reduction of more than $90 \%$.

Given the requirement for a finite frequency mismatch $\Delta f$ in a conventional gyroscope system, the 2-DOF sense-mode architecture can actually improve both robustness and performance. In Fig. 7, the frequency responses of a 2-DOF sense-mode gyroscope and a conventional 1-DOF sense-mode gyroscope with $\Delta f=100 \mathrm{~Hz}$ are presented. Both systems have identical drive oscillators, operated at the drive resonant frequency of $2 \mathrm{kHz}$, with a drive amplitude of $10 \mu \mathrm{m}$. In the sense mode, the primary mass $m_{a}$ of 2-DOF oscillator is equal to the mass of the conventional device, and the mass ratio is $m_{a} / m_{b}=20$. The isolated primary and secondary resonant frequencies are $1975 \mathrm{~Hz}$, which locate the flat region at the $2-\mathrm{kHz}$ operation frequency.

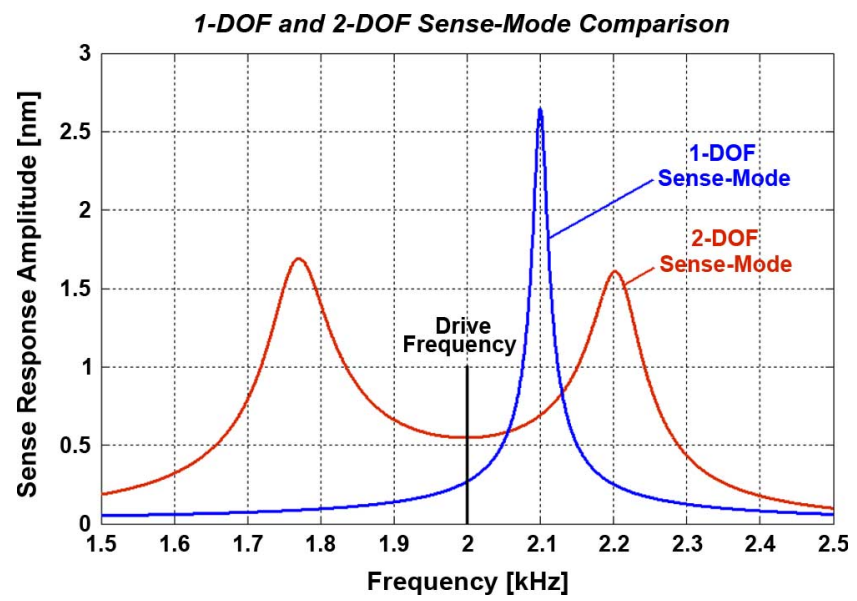

Fig. 7. Comparison of response between the 1-DOF and 2-DOF sense-mode oscillators. The 2-DOF sense-mode oscillator can even improve the scale factor and rate sensitivity compared to a conventional 1-DOF sense-mode oscillator with mismatched modes.

Damping conditions in both devices are also identical, resulting in a $\mathrm{Q}$ factor of 100 in the conventional device.

For $1^{\circ} / \mathrm{s}$ angular rate input, the response amplitude of the 2-DOF system is $0.5480 \mathrm{~nm}$, which is over two times larger than the conventional system response of $0.266 \mathrm{~nm}$ (Fig. 7). When there is a $10 \mathrm{~Hz}$ variation in operation frequency, the response amplitude becomes 0.5492 , changing by less than $0.2 \%$. The same $10 \mathrm{~Hz}$ variation results in $10 \%$ scale factor error in a coventional device with $\Delta f=100 \mathrm{~Hz}$. Thus, the 2-DOF system provides over two times larger scale factor and fifty times higher robustness to $10-\mathrm{Hz}$ frequency variation.

The scale factor of the conventional system becomes higher than the 2-DOF sense system when the $\Delta f$ is less than $50 \mathrm{~Hz}$ in this example. For this range of $\Delta f$, the scale factor error for $10 \mathrm{~Hz}$ variation is more than $16 \%$. Thus, in applications that require higher robustness or fabrication processes with larger relative frequency variation, the 2-DOF sense-mode gyroscope system achieves better performance and robustness.

2) Bandwidth: The mechanical bandwidth of a multi-DOF device is defined by the frequencies at which the amplitude of the sense-mode frequency response increases by $3 \mathrm{~dB}$ versus the minimum value between the peaks, which gives

$$
\begin{aligned}
\omega_{3 \mathrm{~dB} 1,2}^{2}= & \omega_{d}^{2}+(1+\sqrt{2})\left(\frac{\Delta}{2}\right)^{2} \\
& \mp \sqrt{\left(1-\frac{\sqrt{2}}{2}\right) \omega_{d}^{2}+2(1-\sqrt{2})\left(\frac{\Delta}{2}\right)^{4}}
\end{aligned}
$$

where $\omega_{3 \mathrm{~dB} 1}$ and $\omega_{3 \mathrm{~dB} 2}$ are the lower and higher $3 \mathrm{~dB}$ frequencies, respectively. Together, the $3 \mathrm{~dB}$ points from (17) define the bandwidth of the 2-DOF sense system according to $\mathrm{BW}_{3 \mathrm{~dB}}=\omega_{3 \mathrm{~dB} 2}-\omega_{3 \mathrm{~dB} 1}$. If the peak spacing is again assumed to be much smaller than the operational frequency $\omega_{d} \gg \Delta$, the bandwidth expression simplifies to

$$
\mathrm{BW}_{3 \mathrm{~dB}}=\left(\sqrt{1-\frac{\sqrt{2}}{2}}\right) \Delta \approx 0.54 \Delta
$$




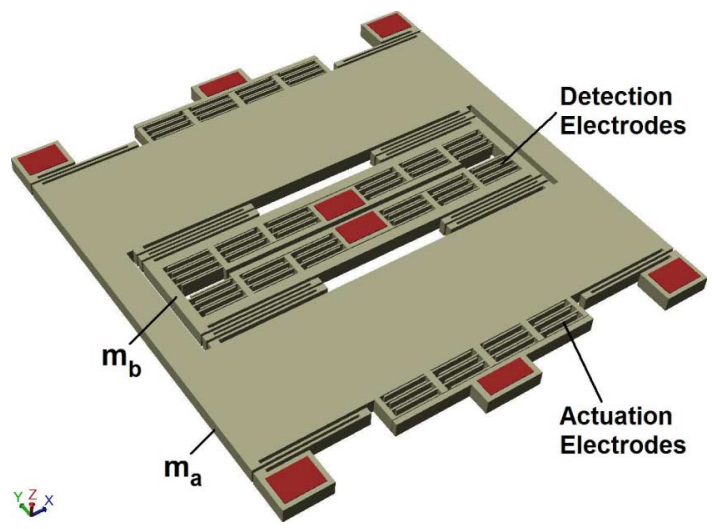

Fig. 8. 2-DOF oscillator test structure, with actuation electrodes attached to the primary mass.

which corresponds to a sense-mode 3-dB bandwidth of roughly half of the peak spacing independent of operational frequency.

\section{Demonstration of 2-DOF OSCILlator Robustness}

In order to characterize parametric sensitivity of the 2-DOF oscillator system frequency response, a prototype gyroscope structure with actuation electrodes attached to the primary mass $m_{a}$ was designed and fabricated (Fig. 8). The primary mass actuation electrodes accurately emulate the Coriolis force generated by the primary mass.

The frequency response of the 2-DOF oscillator was electrostatically detected under varying pressure and temperature conditions in an microminiature refrigerator (MMR) vacuum probe station. The response signal was acquired using off-chip transimpedance amplifiers connected to an Hewlett-Packard Signal Analyzer in sine-sweep mode. Two-port actuation and detection was utilized, where one probe was used to impose the $\mathrm{dc}$ bias voltage on the gyroscope structure through the anchor, one probe was used to apply the ac drive voltage on the actuation port attached to the primary mass $m_{a}$, and the detection port on the secondary mass $m_{b}$ was directly connected to the transimpedance amplifier.

Fig. 9 presents the experimentally measured amplitude and phase responses of the sense-mode passive mass at 5,15 , and 30 Torr. The change in the response amplitude in the flat operating region was observed to be insignificant, while the amplitude around the two resonance peaks increased with decreasing pressures. Furthermore, the phase of the second sense mass was observed to stay constant in the operating frequency band, while the phase changes were observed at the two resonance peaks. Thus, it is experimentally verified that amplitude and phase are unaffected by damping in the flat operating region, in contrast to the abrupt changes around the resonance peaks as in the conventional gyroscopes.

The sensitivity of the prototype gyroscopes to temperature variations was characterized by heating the vacuum chamber of the MMR probe station and continuously monitoring the temperature of the sample using a solid-state temperature sensor attached to the handle wafer. Fig. 10 presents the capacitively
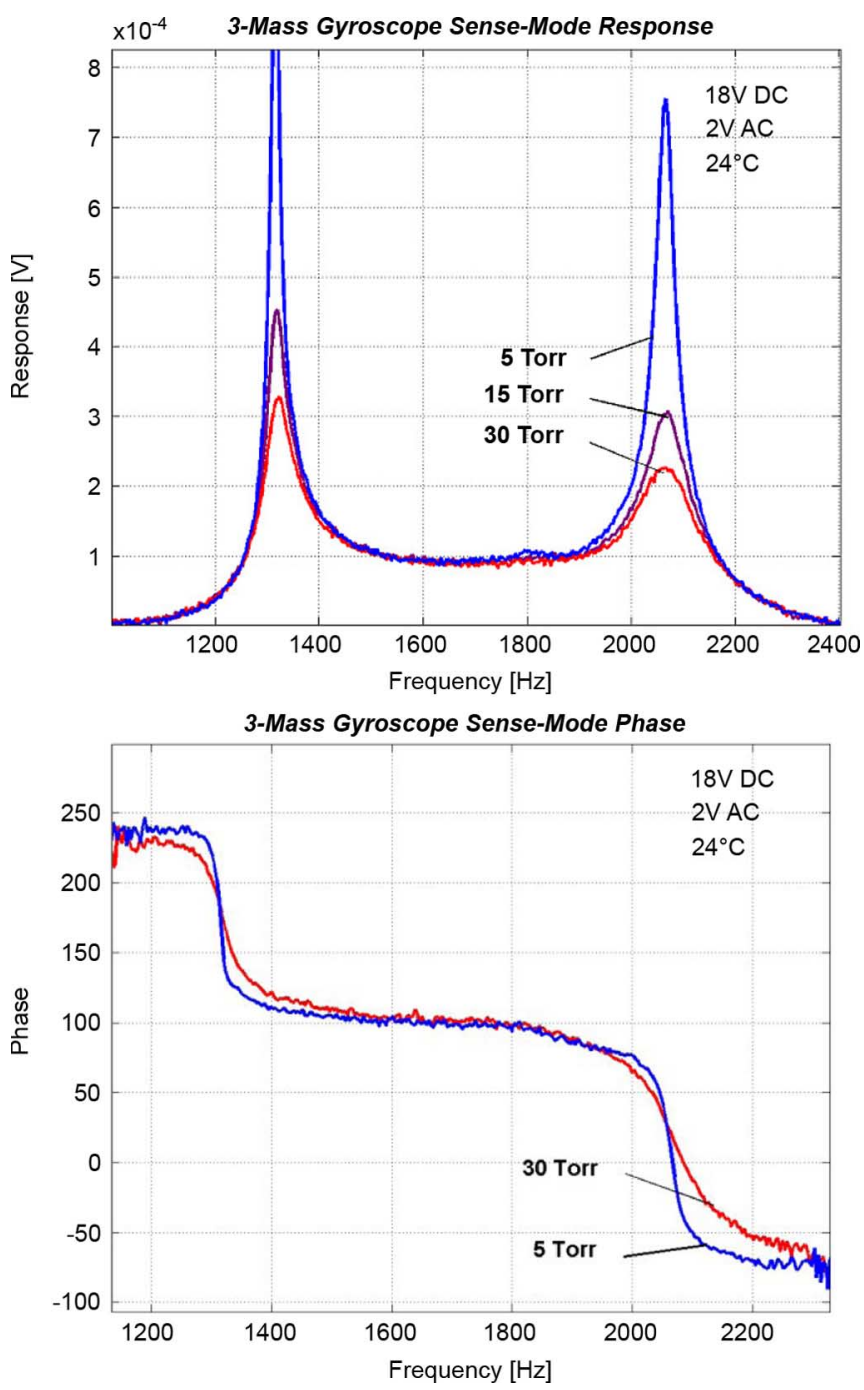

Fig. 9. Electrostatically acquired amplitude and phase response, with changing pressure values. The amplitude and phase of the response remains constant within the flat region.

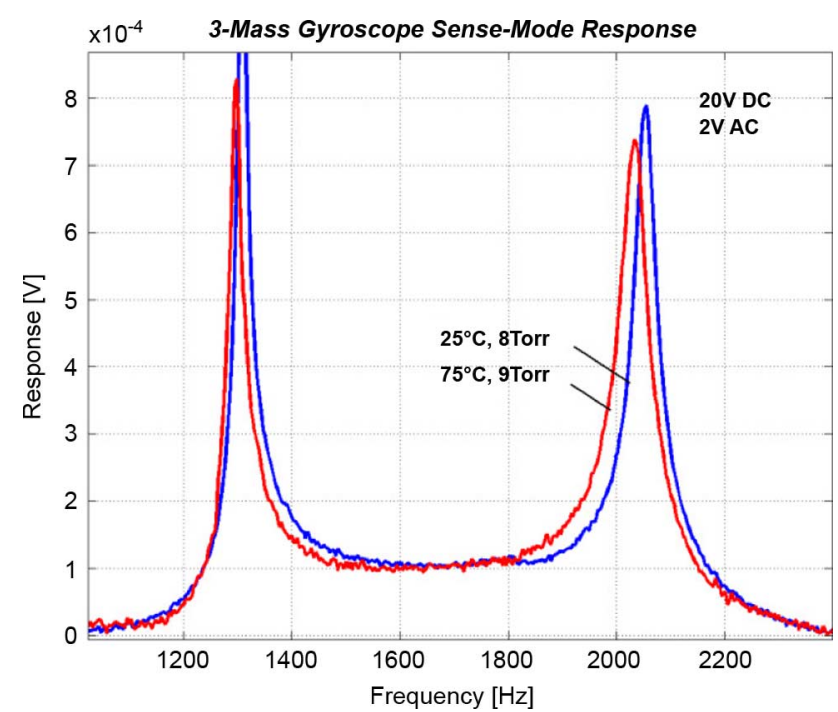

Fig. 10. Frequency response of the sense-mode passive mass, at $25^{\circ} \mathrm{C}$ and $75^{\circ} \mathrm{C}$. The response gain within the flat operating region is observed to stay constant. 


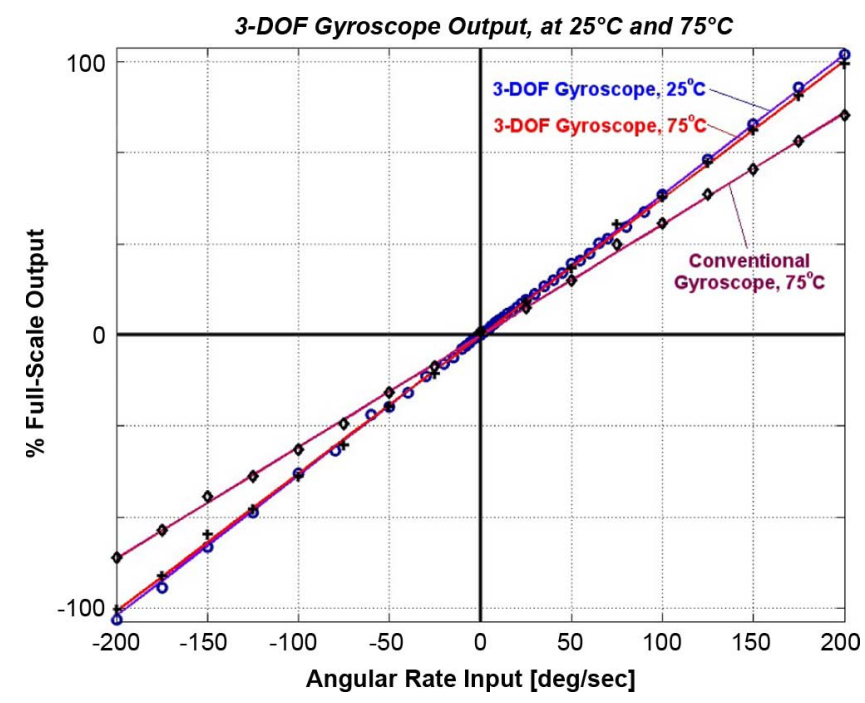

Fig. 11. Measured angular-rate response of the 3-DOF gyroscope and a conventional gyroscope at $25^{\circ} \mathrm{C}$ and $75^{\circ} \mathrm{C}$. Sensitivity of the conventional gyroscope was scaled to match $3-\mathrm{DOF}$ gyroscope at $25^{\circ} \mathrm{C}$.

acquired frequency response of the sensing element at the temperatures $25^{\circ} \mathrm{C}$ and $75{ }^{\circ} \mathrm{C}$. The response amplitude in the flat operating region was observed to be less than $2 \%$ for the $50{ }^{\circ} \mathrm{C}$ variation in temperature, experimentally verifying the improved robustness against temperature variations.

In order to verify that robustness to parameter variations is achieved in the overall Coriolis response, the 2-DOF sensemode gyroscope structure was characterized on the rate table in a thermally controlled chamber. The operation frequency was manually adjusted to track the drive-mode resonance at each temperature. In the 2-DOF sense-mode gyroscope, a temperature variation from $25{ }^{\circ} \mathrm{C}$ to $75^{\circ} \mathrm{C}$ was observed to result in only $1.62 \%$ change in the scale factor, verifying the improved robustness. At elevated temperatures, the linearity of the response was also observed to be preserved. For comparison, a conventional gyroscope fabricated in the same process was tested under identical conditions. A temperature increase from $25{ }^{\circ} \mathrm{C}$ to $75{ }^{\circ} \mathrm{C}$ was observed to result in $19.8 \%$ sensitivity change in the conventional gyroscope, which is more than 12.2 times larger than in the 2-DOF sense-mode gyroscope approach (Fig. 11).

\section{Multi-DOF SENSE-MOdE TUNING Fork Gyroscope}

In this section, we review a second multi-DOF concept that was introduced to address another common challenge for automotive devices: ambient vibrations and shock [8], [11]. A common design technique used to alleviate these issues is the use of tuning fork architectures, which allows the sensor to mechanically reject common mode inputs while preserving the angular rate signal [5]. Since the multi-DOF concept presented in the previous section also used mechanical design to improve temperature robustness, a hybrid device promised a sensor with the benefits of both concepts. Thus, the multi-DOF sense-mode tuning fork gyroscope was introduced intending to combine the robustness of multi-DOF sense systems with the common-mode rejection capability of tuning fork designs [17], [18].

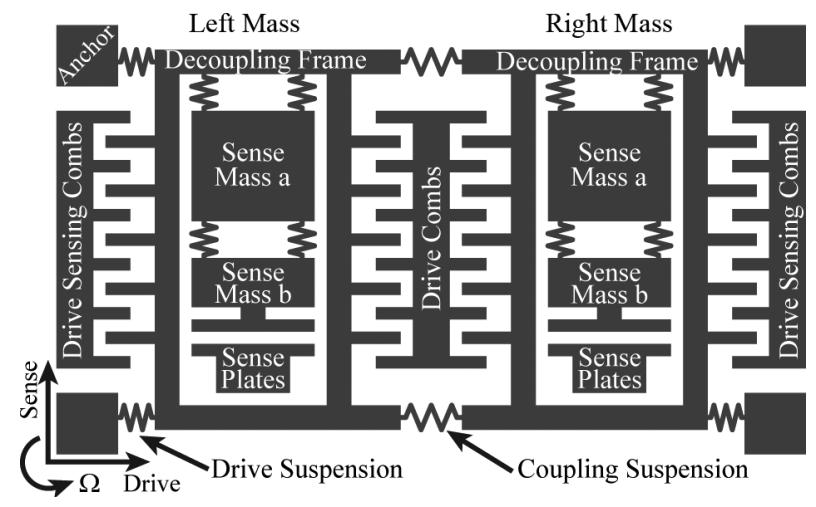

Fig. 12. Schematic of the multi-DOF sense-mode tuning fork gyroscope.

\section{A. Design Concept}

A schematic of the multi-DOF sense-mode tuning fork gyroscope concept is shown in Fig. 12. It consists of two identical multi-DOF systems, labeled left mass and right mass, connected via a coupling suspension forming a 2-DOF dynamic system in the drive mode. These systems are equivalent to the tines of a tuning fork; the major difference between conventional tuning fork gyroscopes [4], [8], however, is that both tines are multi-DOF devices, which contain decoupled 2-DOF sense modes. The frequency response of an ideal multi-DOF tine is identical to the regular multi-DOF gyroscope, Fig. 5, except that the operational frequency of the tuning fork device is the antiphase natural frequency of the 2-DOF drive mode.

The operation of the multi-DOF sense-mode tuning fork, as with conventional ones, is based upon antiphase motion of the drive masses. By driving in antiphase, the Coriolis responses of the corresponding sense systems will also be in antiphase. Thus, a differential of the sense signals will preserve the angular rate signal while rejecting common mode inputs such as vibration and shock. In order to excite the antiphase mode, a central driving electrode, Fig. 12, is used to electrostatically force both the left and right masses simultaneously so that the ideal, linear time invariant equations of motion for the drive mode become

$$
\begin{aligned}
& M_{\delta} \ddot{x}_{L}+c_{\delta} \dot{x}_{L}+\left(k_{\delta}+k_{c}\right) x_{L}-k_{c} x_{R}=-F\left(\omega_{d}\right) \\
& M_{\delta} \ddot{x}_{R}+c_{\delta} \dot{x}_{R}+\left(k_{\delta}+k_{c}\right) x_{R}-k_{c} x_{L}=+F\left(\omega_{d}\right)
\end{aligned}
$$

where $x_{L}$ and $x_{R}$ denote the displacements of the left and right masses, respectively, in the drive direction.

Each drive mass $M_{\delta}=m_{f}+m_{a}+m_{b}$ consists of the decoupling frame $m_{f}$ and both sense masses $m_{a}$ and $m_{b}$ moving in unison along opposite drive directions due to the electrostatic forcing $F\left(\omega_{d}\right)$ at the operational frequency. Each mass is suspended relative to the anchor via the drive suspension $k_{\delta}$, while the coupling suspension $k_{c}$ connects the two tines forming the 2-DOF drive-mode dynamic system. These two stiffnesses determine the operational frequency of the multi-DOF tuning fork

$$
\omega_{d}^{2}=\frac{k_{\delta}+2 k_{c}}{M_{\delta}} .
$$

Despite having different drive modes (conventional 1-DOF versus 2-DOF antiphase operation), the sense modes of the multi-DOF sense-mode tuning fork are exactly the same as the 


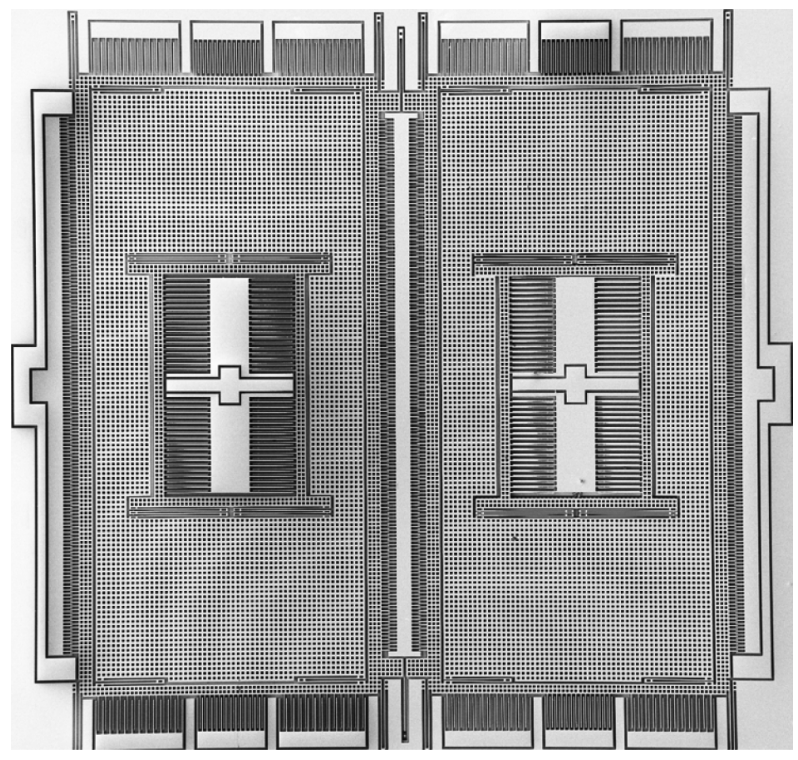

Fig. 13. SEM image of fabricated anti-phase 2-DOF sense-mode gyroscope.

regular multi-DOF device presented in the previous section. While the antiphase forcing provides mechanical rejection of common mode inputs, the multi-DOF sense modes inherently provides the device with improved bandwidth and temperature robustness. As shown in Fig. 5, the sense-mode resonant frequencies are designed to be symmetric about the operational frequency, which in this case, is the antiphase natural frequency as defined in (20). The region between the sense mode resonances not only provides a region of constant amplitude, but also provides a region of constant phase. This allows for easier phase matching of the sense modes, which enhances the ability of the sensor to reject common modes.

\section{B. Common Mode Rejection}

The common-mode rejection capability of the multi-DOF sense-mode tuning fork gyroscope was demonstrated using a shock input applied along the sense mode at atmospheric pressure while monitoring the response of both sense systems. No ac driving voltages were used; however a $10-\mathrm{V}$ dc probing bias was applied to the gyroscope mass for detection.

Fig. 14 (top) shows the experimentally obtained time histories of each sense system triggered due to a mechanical impulse applied at $0.002 \mathrm{~s}$. The outputs track each other, demonstrating that the masses indeed responded in a common mode, as expected. The differential signal is shown in Fig. 14 (bottom), which results in an uncalibrated $12 \mathrm{~dB}$ reduction in amplitude versus the individual signals. The amount of common mode rejection can be further increased by using more precise amplification or capacitance matching between the two outputs [18].

\section{Coriolis Response}

Having demonstrated the common mode response due to shock inputs, the multi-DOF tuning fork gyroscope was then characterized using constant angular rates to verify the antiphase nature of the Coriolis response. This was accomplished by monitoring the sense signal of each mass individually, so
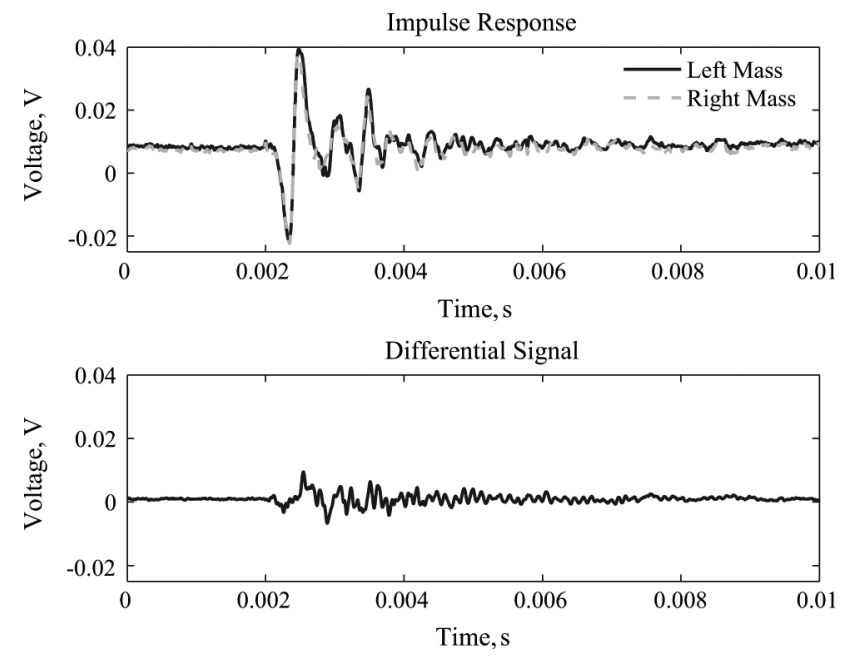

Fig. 14. Experimentally obtained time history of right and left sense mode outputs due to shock input.

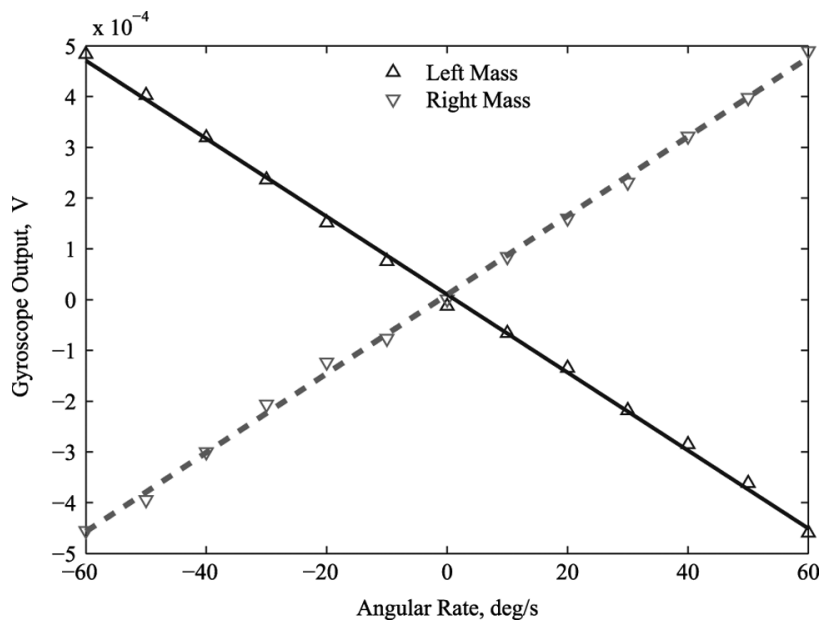

Fig. 15. Constant rate response of both right and left masses of the multi-DOF tuning fork gyroscope.

that the scale factors of each could be observed; the final device output, however, would be the difference of these two signals.

The response of the left and right masses to constant angular rates is presented in Fig. 15. The scale factors were found to be $-7.68 \mu \mathrm{V} / \% / \mathrm{s}$ [linearity of $2.2 \%$ full-scale output (FSO)] for the left mass and $7.78 \mu \mathrm{V} /{ }^{\circ} / \mathrm{s}$ (linearity of $2.2 \% \mathrm{FSO}$ ) for the right mass. As expected, the Coriolis responses of the two masses are in antiphase as demonstrated by the difference in sign of the scale factors [17]. Thus, the presented multi-DOF concept preserves the common-mode rejection capability of tuning fork devices.

\section{Multi-DOF Sense-Mode Gyroscope With Flexible SELECTION OF OPERATIONAL PARAMETERS}

Even though common mode rejection greatly enhances the vibration immunity, operating above the automotive vibration spectrum that extends up to $5 \mathrm{kHz}$ is essential. In this section, we review an alternative MEMS vibratory rate gyroscope design with 2-DOF sense mode recently introduced in [19], which increases the operational frequencies independent of the sense-mode peak separation. The proposed architecture utilizes 


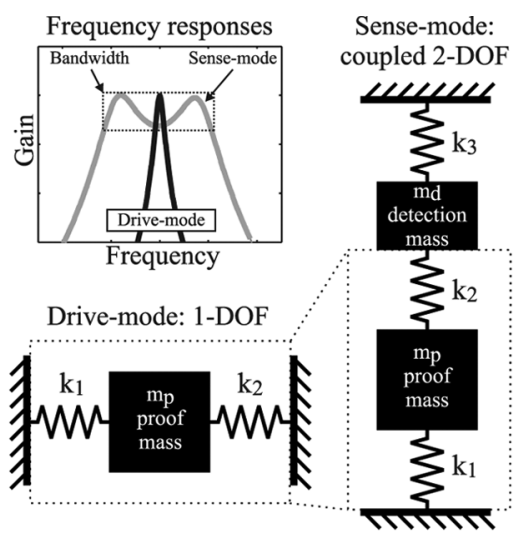

(a) Lumped models of the drive and sense modes.

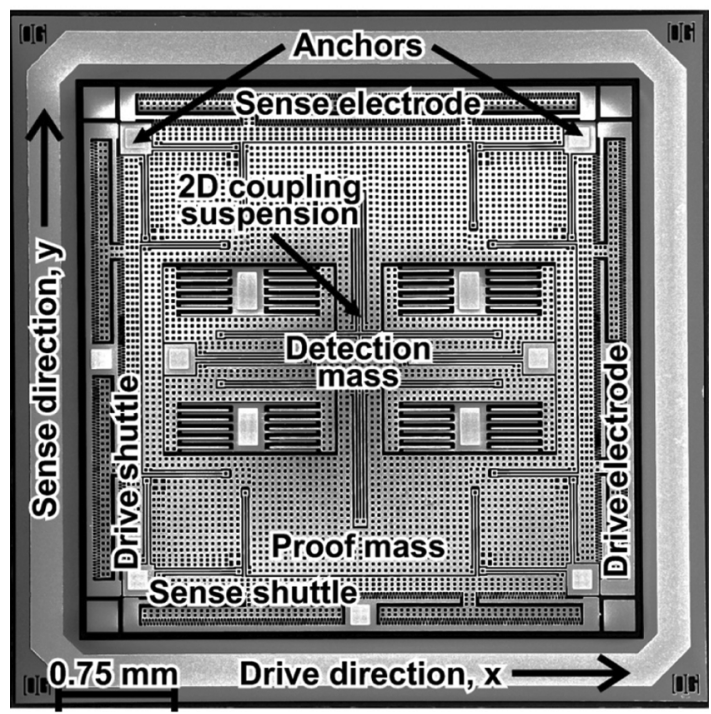

(b) SEM image of a fabricated gyroscope.

Fig. 16. Proposed sensor element with 1-DOF drive mode and a fully coupled 2-DOF sense mode.

a third spring to obtain a fully coupled 2-DOF sense-mode, as in Fig. 16(a), and yields robust, wide-bandwidth devices without sacrifice in response gain. Due to the flexibility of the extended design space of the architecture, the sense-mode resonant peak spacing and bandwidth can be adjusted independently of the operational frequency, die size, and mass ratio. For arbitrary given proof mass $m_{p}$ and detection mass $m_{d}$, the three design stiffnesses are obtained as

$$
\left\{\begin{array}{l}
k_{1}=m_{p} \Phi^{2}-k_{2} \\
k_{2}=\Delta \Phi \sqrt{m_{p} m_{d}} \sqrt{\Phi^{2}-0.25 \Delta \Phi^{2}} \\
k_{3}=m_{d} \Phi^{2}-k_{2}
\end{array}\right.
$$

where $\Phi$ and $\Delta \Phi$ are, respectively, the desired operational frequency and the sense-mode resonant peak spacing. Inherent to the design, the drive mode resonance is automatically positioned between the sense mode peaks, eliminating the need to tune the drive and sense mode frequencies.

A micromachined implementation of the proposed gyroscope is shown in Fig. 16(b). The device consists of an anchored outerframe, two drive mode and two sense mode shuttles, a proof mass $m_{p}$, a detection mass $m_{d}$, and a central anchor. Each of the two drive mode and two sense mode shuttles is suspended relative to the fixed frame by two springs constraining shuttles' mo-

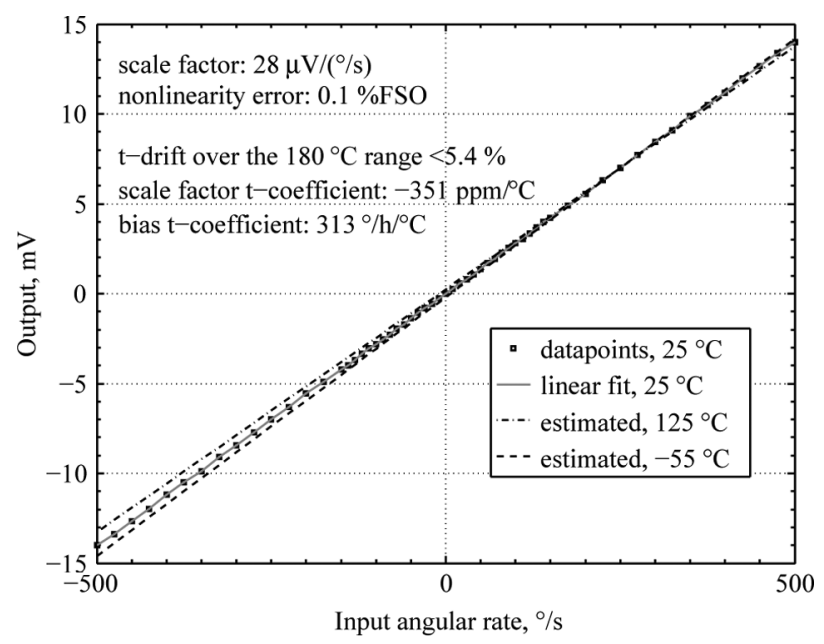

(a) Rate responses at different temperatures.

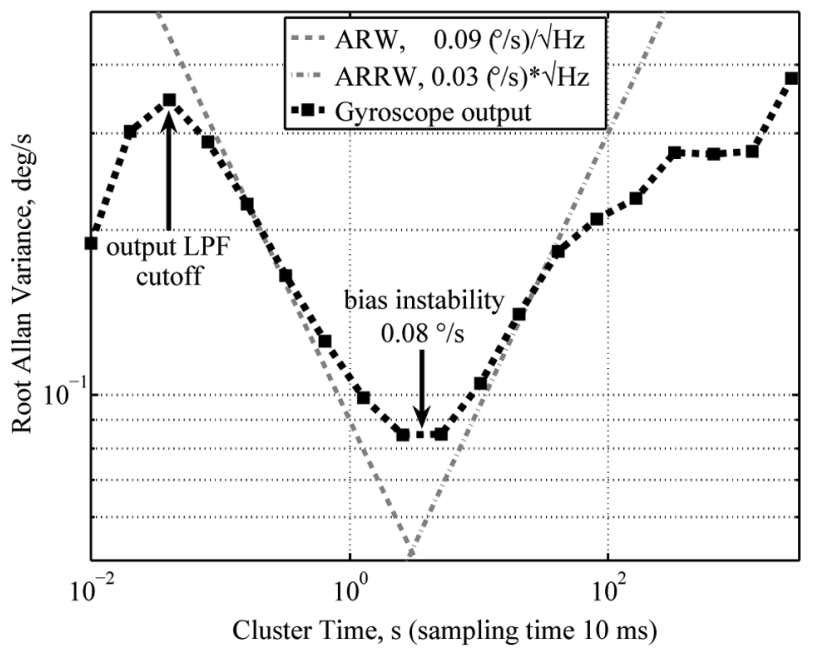

(b) Allan Variance characterization of noise modes at zero rate.

Fig. 17. Experimental characterization of the gyroscope.

tion to the respective axes. Similar suspension elements couple the four shuttles to the proof mass $m_{p}$, forming a symmetrically decoupled suspension [20]. Using the capacitive electrodes on the drive mode shuttles, the proof mass $m_{p}$ is driven into a drive mode oscillation to form a $z$ axis sensitive Coriolis element. Unlike the conventional case [21], [22], the Coriolis-induced motion is not directly picked up from the proof mass $m_{p}$; instead, the proof mass is coupled to the detection mass $m_{d}$ using a bidirectional flexure with equal $x$ and $y$ stiffnesses. The detection mass is also coupled to the substrate with an inner suspension. During rotation, the Coriolis acceleration of the proof mass is transferred to the detection mass, which responds in a wide frequency bandwidth due to the coupled dynamics of the proposed 2-DOF sense mode.

Fabricated prototypes were packaged and characterized under ambient pressure conditions. The measured drive mode resonance was at $2.58 \mathrm{kHz}$, located in-between the 2-DOF sensemode resonances at 2.47 and $2.73 \mathrm{kHz}$. The 2-DOF sense-mode exhibited a $250-\mathrm{Hz} 3-\mathrm{dB}$ bandwidth providing an eight times improvement in temperature drift compared to the identical conventional mode-matched device achieved without sacrifice in gain. Fig. 17(a) shows rate response experimentally obtained 
at room temperature and estimated responses for $-55^{\circ} \mathrm{C}$ and $125^{\circ} \mathrm{C}$ temperatures. Analysis of the gyroscope's noise performance is shown in Fig. 17(b). With the off-chip detection electronics, the measured resolution was $0.09^{\circ} / \mathrm{s} / \sqrt{\mathrm{Hz}}$, and the bias drift was $0.08^{\circ} / \mathrm{s}$.

For the described gyroscope design architecture, the resonant peak spacing of the fully coupled 2-DOF sense mode defines the bandwidth of the gyroscope and can be adjusted independently of the operational frequency and device size. The rate sensitivity and quadrature of the gyroscope are comparable to the best reported performance characteristics for MEMS gyroscopes operated in air, e.g., [23]. At the same time, the gyroscope provides much larger bandwidth than the state of the art rate gyroscopes, as well as excellent robustness to fabrication imperfections and in-operation temperature variations. Similar to the gyroscope design described in Section VI, the proposed device can be utilized in a tuning fork architecture for mechanical rejection of common mode accelerations. Alternatively, due to the flexible scaling, the operational frequency can be increased above the vibrational spectrum.

\section{CONCLUSION}

Automotive applications require low-cost and high-yield devices to meet strict performance specifications under quite demanding environmental conditions. In this paper, we reviewed micromachined gyroscope structural architectures with multi-DOF sense-mode oscillators, that provide inherent robustness against process and environmental variations at the mechanical sensing element level. We first presented the basic concept with a 2-DOF sense mode and a 1-DOF drive mode and illustrated that the 2-DOF architecture is actually capable of improving the mechanical angular rate sensitivity, as well as robustness compared to a conventional mode-mismatched approach. We then introduced the antiphase tuning fork gyroscope with multi-DOF sense modes that utilizes common mode rejection to improve vibration and shock immunity. We finally presented a new multi-DOF concept that allows to increase the operational frequencies above the vibration spectrum while preserving a small sense-mode peak separation. This design trend in the multi-DOF concept is expected to lead to reliable, robust, and high performance-angular-rate sensors with low-production costs and high yields, ideal for the demanding automotive environment.

\section{REFERENCES}

[1] C. Acar and A. Shkel, "MEMS vibratory gyroscopes-Structural approaches to improve robustness," in MEMS Reference Shelf Series. New York: Springer, 2009, ISBN: 978-0-387-09535-6.

[2] MEMS in the Automotive Industry. Market Analysis Report, 2007. [Online]. Available: http://www.wtc-consult.com

[3] A. Madni and L. Costlow, "A third generation, highly monitored, micromachined quartz rate sensor for safety-critical vehicle stability control," in Proc. IEEE Aerosp. Conf. , 2001, vol. 5, pp. 2523-2534.

[4] A. Madni, L. Costlow, and M. Smith, "The $\mu$ Gyro: A quartz MEMS automotive gyroscope," in presented at the 2006 SAE World Congr., Detroit, MI, Apr. 3-6, 2006.

[5] M. Weinberg and A. Kourepenis, "Error sources in in-plane silicon tuning-fork MEMS gyroscopes," J. Microelectromech. Syst., vol. 15, pp. 479-491, Jun. 2006.
[6] U.-M. Gomez, B. Kuhlmann, J. Classen, W. Bauer, C. Lang, M. Veith, E. Esch, J. Frey, F. Grabmaier, K. Offterdinger, T. Raab, H.-J. Faisst, R. Willig, and R. Neul, "New surface micromachined angular rate sensor for vehicle stabilizing systems in automotive applications," in Proc. Int. Conf. Solid-State Sensors, Actuators and Microsyst. (TRANSDUCERS 2005), , vol. 1, pp. 184-187.

[7] R. Neul, U. Gomez, K. Kehr, W. Bauer, J. Classen, C. Doring, E. Esch, S. Gotz, J. Hauer, B. Kuhlmann, C. Lang, M. Veith, and R. Willig, "Micromachined gyros for automotive applications," in Proc. IEEE Sensors, 2005, p. 4.

[8] R. Neul, U.-M. Gomez, K. Kehr, W. Bauer, J. Classen, C. Doring, E. Esch, S. Gotz, J. Hauer, B. Kuhlmann, C. Lang, M. Veith, and R. Willig, "Micromachined angular rate sensors for automotive applications," IEEE Sensors J., vol. 7, pp. 302-309, Feb. 2007.

[9] J. Geen, "Progress in integrated gyroscopes," in Proc. Position Location Navigat. Symp. (PLANS 2004), , pp. 1-6.

[10] J. Geen, "Very low cost gyroscopes," in Proc. IEEE Sensors, 2005, p. 4.

[11] J. A. Geen, S. J. Sherman, J. F. Chang, and S. R. Lewis, "Single-chip surface micromachined integrated gyroscope with $50 \mathrm{deg} / \mathrm{h}$ allan deviation," IEEE J. Solid-State Circuits, vol. 37, no. 12, pp. 1860-1866, Dec. 2002.

[12] R. Eley, M. Durston, C. Fell, and K. Townsend, "Design and development of a low cost digital silicon MEMS gyroscope," in Proc. ITE Symp. Gyro Technol., Sep. 2005.

[13] C. Acar and A. Shkel, "Inherently robust micromachined gyroscopes with 2-DOF sense-mode oscillator," J. Microelectromech. Syst., vol. 15, pp. 380-387, Apr. 2006.

[14] C. Acar and A. Shkel, "Nonresonant micromachined gyroscopes with structural mode-decoupling," IEEE Sensors J., vol. 3, no. 4, pp. 497-506, Aug. 2003.

[15] C. Acar, A. M. Shkel, L. Costlow, and A. M. Madni, "Inherently robust micromachined gyroscopes with 2-DOF sense-mode oscillator," in Proc. IEEE Sensors, Irvine, CA, USA, Nov. 3, 2005, pp. 664-667.

[16] A. R. Schofield, A. A. Trusov, and A. M. Shkel, "Effects of operational frequency scaling in multi-degree of freedom MEMS gyroscopes," IEEE Sensors J., vol. 8, no. 10, pp. 1672-1680, Oct. 2008.

[17] A. R. Schofield, A. A. Trusov, C. Acar, and A. M. Shkel, "Anti-phase driven rate gyroscope with multi-degree of freedom sense mode," in Proc. Int. Solid-State Sens., Actuators, Microsyst. Conf. (TRANSDUCERS 2007), Lyon, France, Jun. 10-14, , pp. 1199-1202.

[18] A. R. Schofield, A. A. Trusov, and A. M. Shkel, "Multi-degree of freedom tuning fork gyroscope demonstrating shock rejection," in Proc. IEEE Sensors, Atlanta, GA, USA, Oct. 28-31, 2007, pp. $120-123$.

[19] A. A. Trusov, A. R. Schofield, and A. M. Shkel, "New architectural design of a temperature robust MEMS gyroscope with improved gainbandwidth characteristics," in Proc. Hilton Head Workshop in Solid State Sensors, Actuators, and Microsyst., Jun. 1-5, 2008.

[20] M. S. Kranz and G. K. Fedder, "Micromechanical vibratory rate gyroscope fabricated in conventional CMOS," in Proc. Symp. Gyro Technol., Stuttgart, Germany, Sep. 16-17, 1997, pp. 3.0-3.8.

[21] S. Alper and T. Akin, "A single-crystal silicon symmetrical and decoupled MEMS gyroscope on an insulating substrate," J. Microelectromech. Syst., vol. 14, pp. 707-717, Aug. 2005.

[22] J. G. L. W.-T. Sung, S. Sung, and T. Kang, "Design and performance test of a MEMS vibratory gyroscope with a novel AGC force rebalance control," J. Micromech. Microeng., vol. 17, no. 10, pp. 1939-1948, Oct. 2007.

[23] S. Alper, K. Azgin, and T. Akin, "A high-performance silicon-on-insulator MEMS gyroscope operating at atmospheric pressure," Sens. Actuators A: Phys., vol. 135, no. 1, pp. 34-42, Mar. 2006.

[24] C. Acar and A. Shkel, "Non-resonant micromachined gyroscopes with structural mode-decoupling," U.S. Patent 7377 167, May 27, 2008.

[25] C. Acar and A. Shkel, "Robust micromachined gyroscopes with two degrees of freedom sense-mode oscillator," U.S. Patent 7284 430, Oct. 23, 2007.

[26] C. Acar and A. Shkel, "Non-resonant four degrees-of-freedom micromachined gyroscope," U.S. Patent 6845 669, Jan. 25, 2005. 


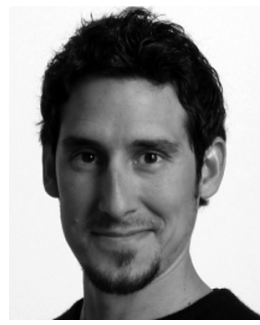

Cenk Acar (M'04) received the B.S. degree in mechanical engineering from Bogazici University, Istanbul, Turkey, and the Ph.D. degree in mechanical and aerospace engineering from the University of California, Irvine, in 2004.

$\mathrm{He}$ joined as a Senior Inertial Microelectromechanical Systems (MEMS) Engineer at the Systron Donner Automotive, Schneider Electric, Concord, $\mathrm{CA}$, where he is currently leading the research and development efforts on the next-generation micromachined inertial sensors product line in the Silicon MEMS Program Manager role. He is the author or coauthor of the book MEMS Vibratory Gyroscopes, and more than 20 journal and international conference publications on MEMS inertial sensors. He is the holder of 15 U.S. and 11 international pending patents. His current research interests include design, modeling, fabrication, characterization and control of MEMS inertial sensors.

Dr. Acar is a member of the American Society of Mechanical Engineers. He has served on the Organizing and Technical Committees of Leading International MEMS Conferences, and is a Reviewer of major MEMS journals.

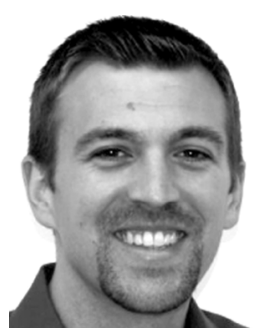

Adam R. Schofield (S'06-M'08) received the B.S. degree (summa cum laude, with honors) in mechanical engineering and the M.S. degree in mechanical engineering (supported by an Ohio Space Grant Fellowship) from the University of Dayton, Dayton, $\mathrm{OH}$, in 2002 and 2005, respectively. He is currently working towards the Ph.D. degree in mechanical and aerospace engineering at the University of California, Irvine.

His current research interests include the design, fabrication, and characterization of microelectromechanical systems inertial sensors.

Mr. Schofield is a member of Pi Tau Sigma, Tau Beta Pi, Golden Key Honor Society, and the American Society of Mechanical Engineers. He was awarded the Class of 2002 Award for Outstanding Mechanical Engineering Achievement by the University of Dayton.

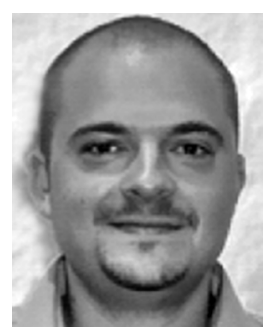

Alexander A. Trusov (S'06) received the B.S degree in applied mathematics and mechanics from Moscow State University, Moscow, Russia, in 2004, and the M.S. degree in mechanical and aerospace engineering from the University of California, Irvine, in 2006. He is currently working towards the $\mathrm{Ph} . \mathrm{D}$. degree at the University of California.

His current research interests include the design, fabrication, and characterization of inertial microelectromechanical systems (MEMS) and the development of novel actuation, detection, signal processing, and control schemes for dynamic MEMS.

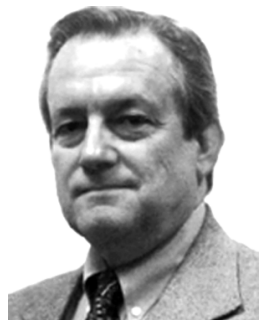

Lynn E. Costlow (M'00) received the B.S.E.E. degree from the University of Texas, Austin, and the M.S.E.E. degree from the Southern Methodist University, Dallas, TX.

$\mathrm{He}$ is the recently retired Director of Engineering for Systron Donner Automotive, Custom Sensors and Technologies, Schneider Electric. He has 21 years experience in aircraft fault tolerant flight control system development at Honeywell on many civilian, military, and National Aeronautics and Space Administration (NASA) experimental aircraft. He has been involved in the inertial microelectromechanical systems (MEMS) field since 1992, and for the last 13 years, has managed product development and research in the expanding automotive MEMS rate gyro market for electronic stability control brake systems and rollover prevention.

Mr. Costlow is a member of the American Society of Mechanical Engineers and the Society of Automotive Engineers.

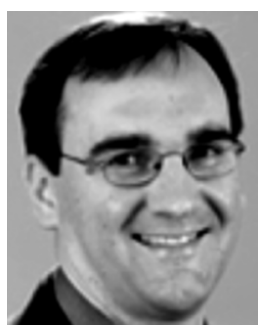

Andrei M. Shkel (S'95-A'98-SM'08) received the Diploma degree (with excellence) in mechanics and mathematics from Moscow State University, Moscow, Russia, in 1991, and the Ph.D. degree in mechanical engineering from the University of Wisconsin, Madison, in 1997.

He was a Postdoctoral Researcher with the Berkeley Sensor and Actuator Center. He was involved in research and consulting positions with several high-tech and venture companies. Since 2000 , he has been a member of the faculty of the University of California, Irvine (UCI), where he is currently an Associate Professor in the Department of Mechanical and Aerospace Engineering, Department of Electrical Engineering and Computer Sciences, and Department of Biomedical Engineering. He is also the Director of the UCI Microsystems Laboratory. He is the holder of 12 U.S. and international patents (with 15 pending) on micromachined angle-measuring gyroscopes, wide-bandwidth rate gyroscopes, design and fabrication of light manipulators and tunable optical filters, and hybrid micromachining processes. His current research interests, which are reflected in more than 100 publications, include solid-state sensors and actuators, microelectromechanical-system-based neuroprosthetics, sensor-based intelligence, and control theory.

Dr. Shkel was a Guest Editor for two Special Issues of the IEEE SENSORS JouRNAl, the General Chair of the 2005 IEEE Sensors Conference, and the Vice General Chair and Publications Chair of the 2002-2006 IEEE Sensors Conferences. He is an Editor of the Journal of Microelectromechanical Systems and the Vice President of the IEEE Sensors Council. He was the recipient of the 2006 Research Award of the UCI School of Engineering, the 2005 National Science Foundation CAREER Award, the 2002 George E. Brown, Jr., Award, and a 2001 Fellowship from the Japanese Advanced Science Institute. 